\title{
Synthesis of graphene on gold
}

Tuba Oznuluer, Ercag Pince, Emre O. Polat, Osman Balci, Omer Salihoglu, and Coskun Kocabas

Citation: Appl. Phys. Lett. 98, 183101 (2011);

View online: https://doi.org/10.1063/1.3584006

View Table of Contents: http://aip.scitation.org/toc/apl/98/18

Published by the American Institute of Physics

\section{Articles you may be interested in}

Graphene segregated on Ni surfaces and transferred to insulators

Applied Physics Letters 93, 113103 (2008); 10.1063/1.2982585

The study of interaction between graphene and metals by Raman spectroscopy

Journal of Applied Physics 109, 07C501 (2011); 10.1063/1.3536670

Plasmon-polaritons on graphene-metal surface and their use in biosensors

Applied Physics Letters 100, 213110 (2012); 10.1063/1.4721453

Solubility and Diffusion Coefficient of Carbon in Nickel: Reaction Rates of Nickel-Carbon Alloys with Barium Oxide

Journal of Applied Physics 23, 1305 (2004); 10.1063/1.1702064

Monolayer graphene growth on $\mathrm{Ni}(111)$ by low temperature chemical vapor deposition

Applied Physics Letters 100, 021601 (2012); 10.1063/1.3675481

Graphene growth on polycrystalline Ru thin films

Applied Physics Letters 95, 133109 (2009); 10.1063/1.3224913

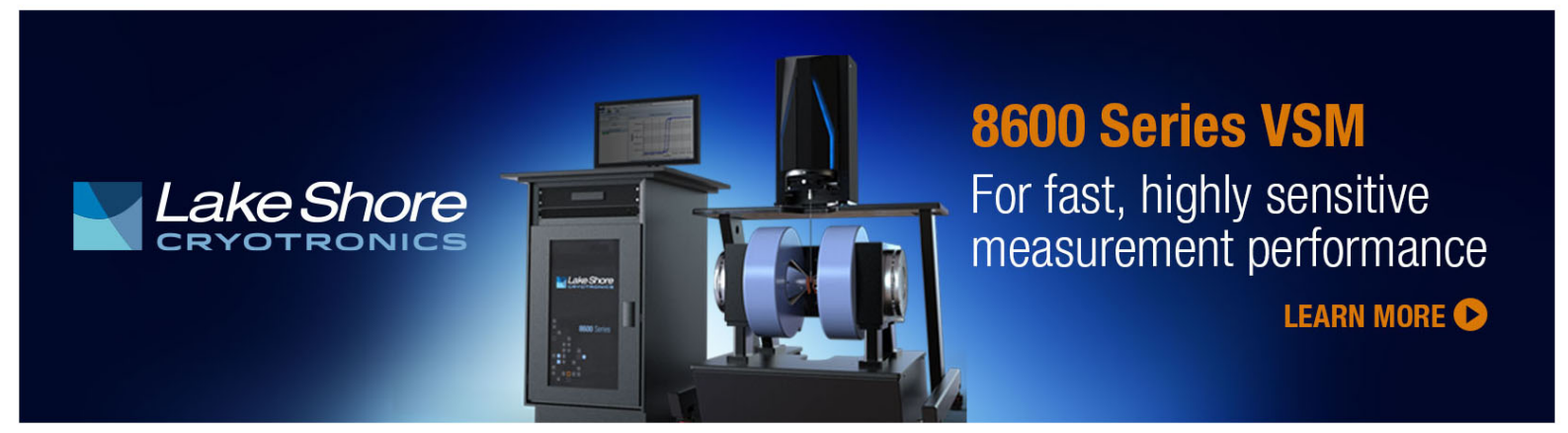




\title{
Synthesis of graphene on gold
}

\author{
Tuba Oznuluer, ${ }^{1}$ Ercag Pince, ${ }^{2}$ Emre O. Polat, ${ }^{2}$ Osman Balci, ${ }^{2}$ Omer Salihoglu, ${ }^{2}$ and \\ Coskun Kocabas ${ }^{2, a)}$ \\ ${ }^{1}$ Department of Chemistry, Ataturk University, 25240 Erzurum, Turkey \\ ${ }^{2}$ Department of Physics, Advanced Research Laboratories, Bilkent University, 06800 Ankara, Turkey
}

(Received 27 January 2011; accepted 9 March 2011; published online 2 May 2011)

\begin{abstract}
Here we report chemical vapor deposition of graphene on gold surface at ambient pressure. We studied effects of the growth temperature, pressure, and cooling process on the grown graphene layers. The Raman spectroscopy of the samples reveals the essential properties of the graphene grown on gold surface. In order to characterize the electrical properties of the grown graphene layers, we have transferred them on insulating substrates and fabricated field effect transistors. Owing to distinctive properties of gold, the ability to grow graphene layers on gold surface could open new applications of graphene in electrochemistry and spectroscopy. (C) 2011 American Institute of Physics. [doi:10.1063/1.3584006]
\end{abstract}

Graphene, the two-dimensional (2D) crystal of $s p^{2}$ hybridized carbon, provides unique electronic ${ }^{1}$ and mechanical properties. ${ }^{2}$ Recent progress on synthesis of graphene on large area substrates ${ }^{3}$ fosters new applications of the 2D crystal. High speed electronics ${ }^{4}$ and macroelectronics ${ }^{5}$ are two examples of these emerging applications. Catalytic decomposition of hydrocarbons on transition metals provides a more promising method for large area growth of high quality graphene layers. Various methods such as chemical vapor deposition (CVD) ${ }^{3}$ surface segregation, ${ }^{6}$ solid carbon source, ${ }^{7}$ and ion implantation ${ }^{8}$ have been used to synthesis of graphene layers on metal surfaces. Nickel ${ }^{9}$ and copper ${ }^{10}$ are the most commonly used metal substrates for the growth. Other metals such as ruthenium, iridium, platinum, palladium, and cobalt have also been used as a substrate for CVD of graphene. ${ }^{10-13}$ Ability to grow graphene on different metals provides opportunities for new applications and more inside to understand the growth mechanism. In this Letter we show a method to directly grow graphene layers on gold surface in various forms such as thin films, foils and wires.

Formation of graphene on metal surfaces has been known for 40 years. ${ }^{13}$ Single crystal Ru and Pt are the earliest metals on which epitaxial graphene due to the segregation of carbon impurities has been observed. ${ }^{13}$ Recent studies have demonstrated CVD of a single layer graphene on large number of metal substrates. Gold surface, however, has not been used for graphene growth. Previously adsorption of graphenelike polycyclic hydrocarbon across gold step edges has been reported. ${ }^{14}$ We believe that the synthesis of large area graphene on gold surface could motivate new applications especially in electrochemistry ${ }^{15,16}$ and spectroscopy. ${ }^{17}$ It is known that gold surface has no catalytic activity. Gold nanoparticles, however, show some diameter dependent catalytic activity for oxidation of carbon monoxide and alcohols. ${ }^{18}$ It is found that the smaller particles are more active for oxidation. Furthermore growing single-walled carbon nanotubes (SWNTs) and multiwalled carbon nanotubes over gold nanoparticles also shows catalytic activity of the gold nanoparticles. Bhaviripudi et al. ${ }^{19}$ grew SWNTs on

\footnotetext{
a) Author to whom correspondence should be addressed. Electronic mail: ckocabas@fen.bilkent.edu.tr.
}

$\mathrm{SiO}_{2}$ substrates using $\mathrm{Au}$ nanoparticles. More recently Yuan et al. ${ }^{20}$ showed the growth of well aligned SWNT using Au particles. This catalytic activity could be due to the solubility of carbon in gold clusters. ${ }^{21}$ The mechanism of catalytic activity of Au particles during CVD process still remains unclear. We believe that the role of gold nanoparticle during the CVD process could provide more inside to understand the growth mechanism of graphene layers on gold surface.

Figure 1 shows the schematic representation of the steps of the preparation of the gold foils, deposition, and transfer process of the graphene layers on dielectric substrates. We used $25 \mu \mathrm{m}$ thick gold foils obtained by pressing high purity gold plates (99.99\% from Vakıf Bank). In order to remove impurities and reconstruct the single crystalline surface, the gold foils were annealed with a hydrogen flame for $20 \mathrm{~min}$ before use. Owing to the fast heating and cooling rates, hydrogen flame annealing provides more complete crystallization of the gold foil than the furnace annealing. ${ }^{22}$ The result of this treatment was a polycrystalline gold surface partially (111) oriented, with a roughness lower than a few nanometers. After the annealing step, the gold foil is placed in quartz chamber and the chamber is flushed with Ar gas for 5 min. The foil is heated up to $975{ }^{\circ} \mathrm{C}$ under $\mathrm{Ar}$ and $\mathrm{H}_{2}$ flow [240 SCCM and 8 SCCM, respectively (SCCM denotes cubic centimeter per minute at STP)]. Methane gas with a rate of flow of 10 SCCM is sent to the chamber for $10 \mathrm{~min}$. After stopping the methane flow, we cooled the chamber with a natural cooling rates around $10 \mathrm{C} / \mathrm{s}$. We have also developed a transfer printing method to the transfer the graphene from the gold foil to insulating dielectric substrates. After the deposition, an elastomeric stamp polydimethylsiloxane (PDMS) is applied on the graphene coated gold foil. The gold layer is etched by diluted gold etchant (type TFA, Transene Co. Inc.). After complete etching of the gold foil, the graphene layer on PDMS is applied on a $100 \mathrm{~nm} \mathrm{SiO}{ }_{2}$ coated silicon wafers. Peeling the PDMS releases the graphene on the dielectric surface.

After the growth, we inspect the grown graphene layers using optical microscope and scanning electron microscope. Figure 2(a) shows scanning electron micrograph of the graphene on gold foils, respectively. The inset in Fig. 2(a) shows the optical micrograph of the graphene on the gold 

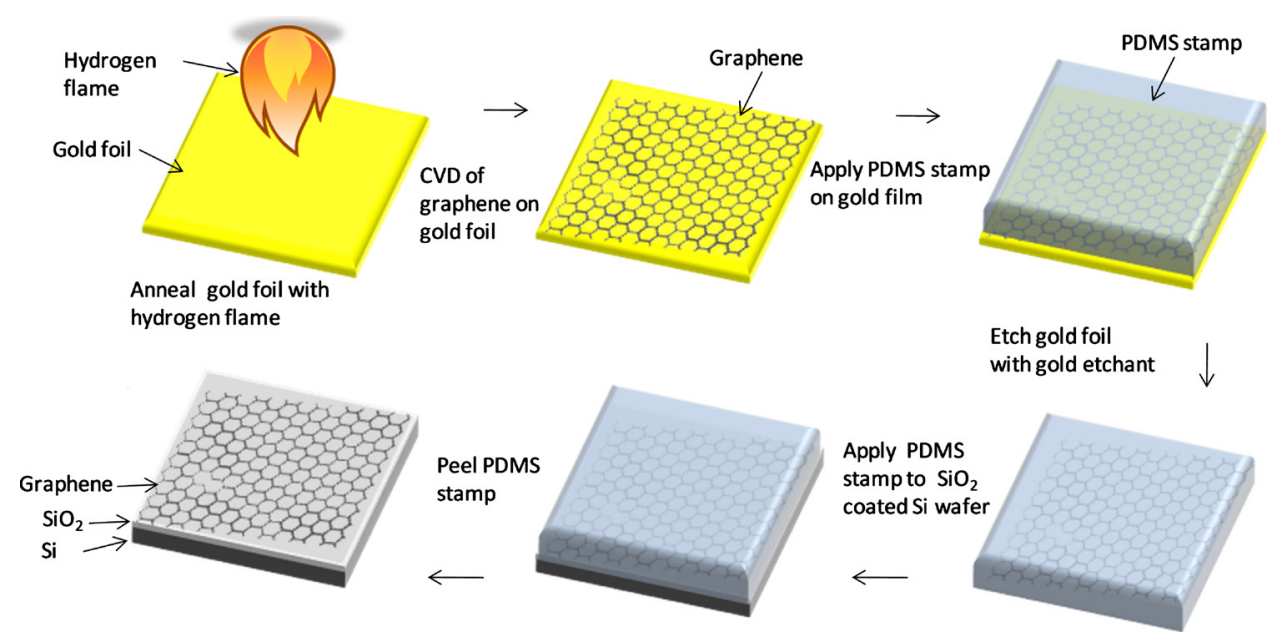

FIG. 1. (Color online) Schematic representation of the steps of deposition and transfer process of graphene growth on gold film. foil. The grain boundaries and wrinkles on the polycrystalline gold surface are clearly seen.

Raman spectroscopy provides clear finger prints of graphene layers. Raman spectrum of the samples was measured using a confocal microraman system (Horiba Jobin Yvon) in back-scattering geometry. A $532 \mathrm{~nm}$ diode laser is used as an excitation source and the Raman signal is collected by a cooled charge-coupled device camera. Figure 2(b) and 2(c) shows the Raman spectrum of the graphene as grown on gold and after the transferring on $\mathrm{SiO}_{2}$ wafer. The expected Raman peaks of D, G, $\mathrm{D}^{\prime}$, and 2D can be seen on the graph. The position of D, G, $\mathrm{D}^{\prime}$, and 2D peaks are 1371, 1600,1640 , and $2743 \mathrm{~cm}^{-1}$ on gold and 1339, 1584, 1620 and $2676 \mathrm{~cm}^{-1}$ on $\mathrm{SiO}_{2}$ substrate. There is a significant redshift after the transfer process, likely due to release of compressive strain on the graphene film. The inset in Fig. 2(c) shows the zoomed 2D peak and the Lorentzian fit. The fit has a symmetric Lorentzian shape with a width of $37 \mathrm{~cm}^{-1}$. The shape and the width of the 2D peak is a good indication of single layer graphene or noninteracting a few layers of graphene. The D-band Raman signal of graphene on gold is relatively more intense than the graphene grown on copper. This intense D band is likely due to the large lattice mismatch between gold and graphene. The lattice constant of bulk graphite and hexagonally closed-packed gold surface are $2.46 \AA$ and $2.88 \AA$, respectively.

In order to find the optimum growth conditions we performed Raman spectroscopy on samples grown at temperatures between 850 and $1050{ }^{\circ} \mathrm{C}$ [see Fig. 2(d)]. At low temperatures around $850{ }^{\circ} \mathrm{C}$, we do not observe a significant 2D peak. On the other hand at very high temperatures there is a significant $\mathrm{D}$ and $\mathrm{G}$ peaks which indicates formation of multilayer defective graphene layers. For the samples grown at temperatures between 850 and $1000{ }^{\circ} \mathrm{C}$, the Raman spectra look relatively the same. We have also studied the effect of the cooling process on the grown graphene layers. However we do not observe any significant change in the Raman spectra for cooling rates between 10 and $0.5 \mathrm{C} / \mathrm{s}$.

Figures 3(a) and 3(b) shows micro-Raman mapping images of the graphene layers on $\mathrm{SiO}_{2}$. The micro-Raman mapping images are measured using confocal microraman system with a spatial resolution of $300 \mathrm{~nm}$. Figure 3(a) shows the 2D map of the Lorentzian width of 2D peak obtained by scanning the sample and Lorentzian curve fitting for each point. The intensity ratio mapping images of $2 \mathrm{D}$ and $\mathrm{G}$ peaks is shown in Fig. 3(b). The histogram of the Lorentzian width and $\mathrm{I}_{2 \mathrm{D}} / \mathrm{I}_{\mathrm{G}}$ peaks are given in Figs. 3(c) and 3(d), respectively. The average width and the intensity ratio is around $40 \mathrm{~cm}^{-1}$ and 1.5 , respectively. The distributions of the histograms further support high percentage of single layer graphene.

To further characterize the grown graphene layers, we have fabricated FETs in thin film geometry. Source and drain electrodes are fabricated on the transferred graphene layer using a standard UV photolithography. The schematic representation of the graphene FET is shown in Fig. 4(a). The devices are isolated from each other by $\mathrm{O}_{2}$ plasma etching of the graphene layers between the devices. A highly doped silicon substrate functions as a global gate electrode and 100 $\mathrm{nm}$ thick $\mathrm{SiO}_{2}$ forms the gate dielectric. The 2D Raman intensity map of the graphene layer used for the device is shown in Fig. 4(b). Figure 4(c) shows the transfer characteristics of a device with a channel length of $8 \mu \mathrm{m}$ and channel width of $100 \mu \mathrm{m}$ at a drain bias of $1 \mathrm{~V}$. The Dirac point of
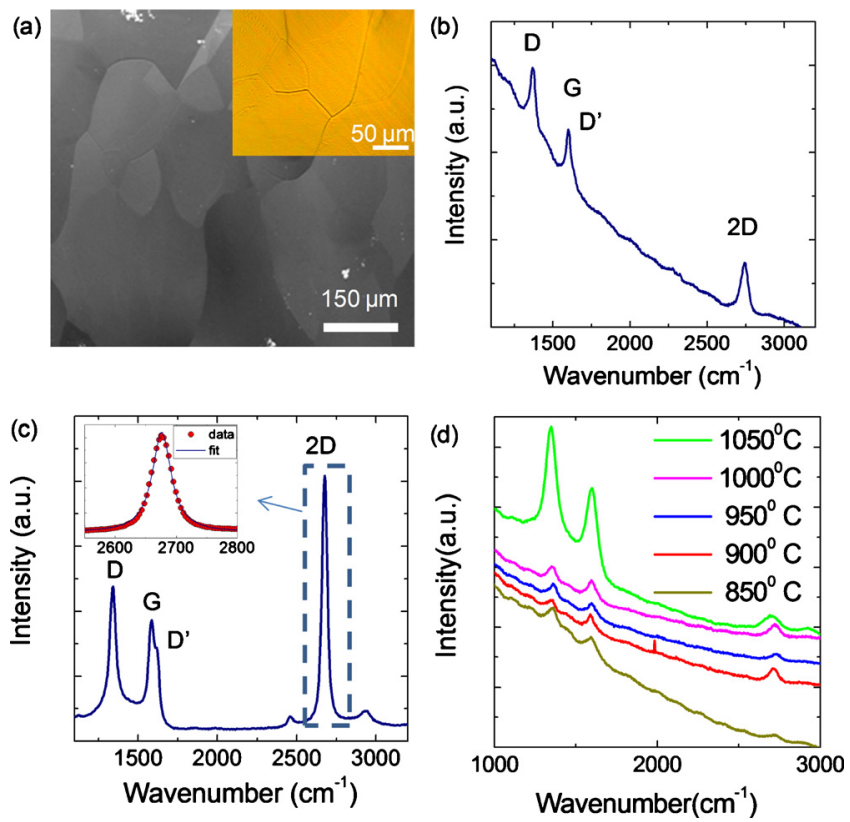

FIG. 2. (Color online) (a) Scanning electron micrograph of the graphene coated gold foils. The inset shows the optical micrograph of the same area (b) Raman spectra of the graphene as grown on gold surface. (c) Raman spectra of the graphene on $\mathrm{SiO}_{2}$ substrate. The inset shows the zoomed spectrum of the 2D peak and Lorentzian fit. The width of the Lorentzian is around $37 \mathrm{~cm}^{-1}$. (d) Raman spectra of the graphene samples grown at a range of temperatures between 850 and $1050{ }^{\circ} \mathrm{C}$. 


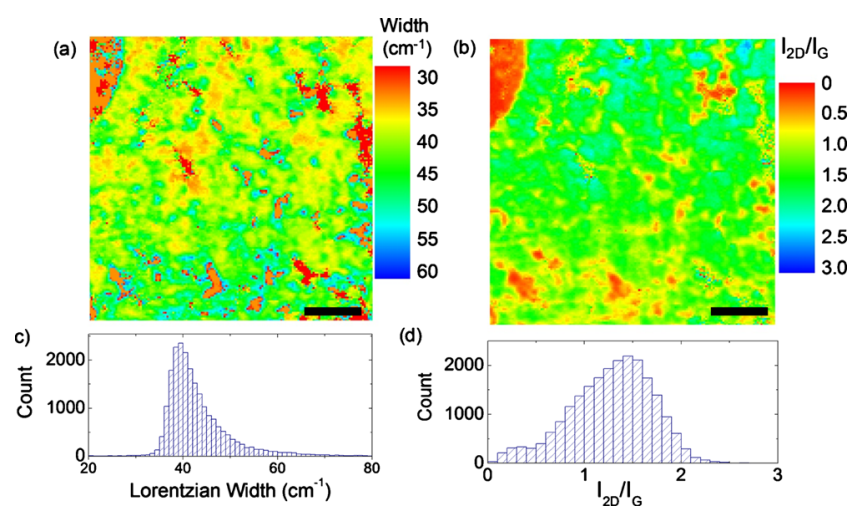

FIG. 3. (Color online) The micro-Raman mapping images of (a) the Lorentzian width of the $2 \mathrm{D}$ peak and (b) intensity ratio $\left(\mathrm{I}_{2 \mathrm{D}} / \mathrm{I}_{\mathrm{G}}\right)$ of $2 \mathrm{D}$ and $\mathrm{G}$ peaks of the graphene on $\mathrm{SiO}_{2}$ layer. The Lorentzian widths are obtained by fitting the spectrum for each point. The scale bars are $5 \mu \mathrm{m}$. Histogram of (c) the Lorentzian width of the $2 \mathrm{D}$ peak and (d) the intensity ratio $\left(\mathrm{I}_{2 \mathrm{D}} / \mathrm{I}_{\mathrm{G}}\right)$.

the device is around $-20 \mathrm{~V}$ which is likely because of the trapped charges on the dielectric layer or unintentional doping during the fabrication process. The on-off ratio of the device is around 2. The calculated field effect mobility of the device is around $20 \mathrm{~cm}^{2} / \mathrm{V} \mathrm{s}$. The output characteristics are given in Fig. 4(d). The modulation of the channel conductivity is clearly seen from the graph.

The Raman spectra together with the transport measurements provide solid evidence that gold surface can be used as a substrate for graphene. Graphene growth on metal substrates shows different growth mechanism depending on the solubility of carbon in the metal. Two different growth mechanisms have been proposed for nickel and copper substrate. Very recently, using sequentially introduced isotopic carbon, Li et al. $^{23}$ demonstrated that the growth mechanism on nickel is based on diffusion and precipitation, however, the growth mechanism on copper is based on surface adsorption. Maximum solubility values of carbon in nickel, copper, and gold are $2.7 \%, 0.04 \%$, and $0.06 \%$, respectively. ${ }^{24}$ The solubility in gold is slightly more than copper and much less than nickel. Based on these solubility values and the observed minor effect of cooling rates, we speculate that the growth mechanism of graphene on gold surface could be similar with copper.

In summary we have reported the synthesis of graphene layers on gold surface using CVD technique. The Raman spectra of the samples reveal the essential feature of the graphene grown on gold. The back-gated FETs that use the transferred graphene layers as an effective semiconductor are fabricated and characterized. Further surface characterization experiments are needed to understand the growth mechanism and the nature of the interaction between the gold and graphene. We believe that, gold surface coated with graphene layer could provide a unique configuration for various new applications ranging from surface plasmon resonance sensors to electrochemical analysis.

This work was supported by the Scientific and Technological Research Council of Turkey (TUBITAK) Grant No. 109T259 and Marie Curie International Reintegration Grant (IRG) Grant No. 256458. (a)

(b)
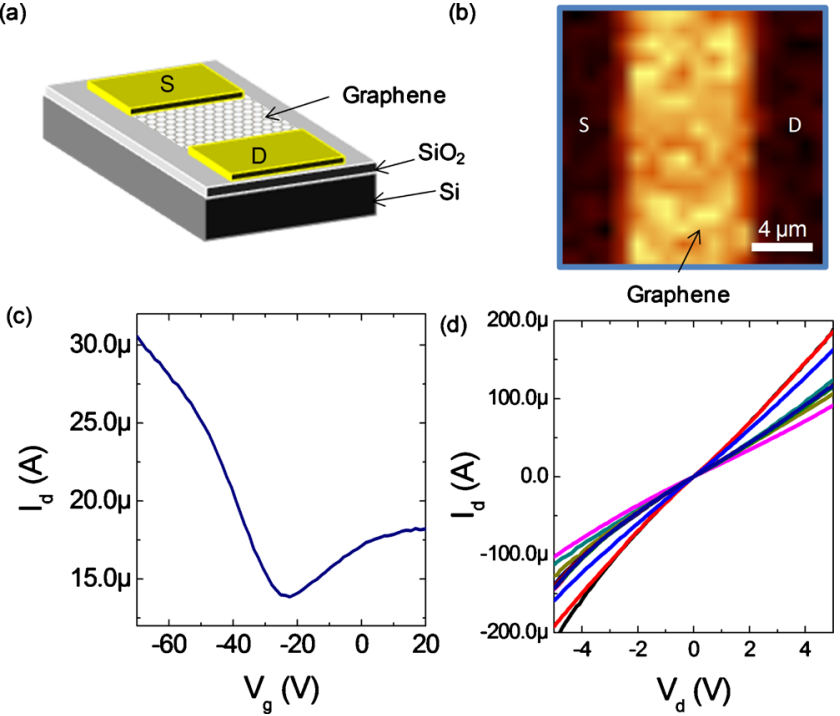

FIG. 4. (Color online) (a) Schematic representation of the back-gated graphene FETs. (b) Raman intensity map of 2D peak from the graphene layer bridging the source and drain electrodes. (c) Transfer and (d) output characteristics the transistor with a channel width of $100 \mu \mathrm{m}$ and a channel length of $8 \mu \mathrm{m}$.

${ }^{1}$ K. S. Novoselov, A. K. Geim, S. V. Morozov, D. Jiang, Y. Zhang, S. V. Dubonos, I. V. Grigorieva, and A. A. Firsov, Science 306, 666 (2004).

${ }^{2}$ A. H. Castro Neto, F. Guinea, N. M. R. Peres, K. S. Novoselov, and A. K. Geim, Rev. Mod. Phys. 81, 109 (2009).

${ }^{3}$ X. S. Li, W. W. Cai, J. H. An, S. Kim, J. Nah, D. X. Yang, R. Piner, A. Velamakanni, I. Jung, E. Tutuc, S. K. Banerjee, L. Colombo, and R. S. Ruoff, Science 324, 1312 (2009).

${ }^{4}$ Y. M. Lin, C. Dimitrakopoulos, K. A. Jenkins, D. B. Farmer, H. Y. Chiu, A. Grill, and P. Avouris, Science 327, 662 (2010).

${ }^{5}$ J. A. Rogers, Nat. Nanotechnol. 3, 254 (2008).

${ }^{6}$ N. Liu, L. Fu, B. Dai, K. Yan, X. Liu, R. Zhao, Y. Zhang, and Z. Liu, Nano Lett. 11, 297 (2011).

${ }^{7}$ Z. Sun, Z. Yan, J. Yao, E. Beitler, Y. Zhu, and J. M. Tour, Nature (London) 468, 549 (2010).

${ }^{8}$ S. Garaj, W. Hubbard, and J. A. Golovchenko, Appl. Phys. Lett. 97, 183103 (2010).

${ }^{9}$ A. Reina, X. Jia, J. Ho, D. Nezich, H. Son, V. Bulovic, M. S. Dresselhaus, and J. Kong, Nano Lett. 9, 30 (2009).

${ }^{10}$ L. Gao, J. R. Guest, and N. P. Guisinger, Nano Lett. 10, 3512 (2010).

${ }^{11}$ J. Coraux, A. T. N'Diaye, M. Engler, C. Busse, D. Wall, N. Buckanie, F. Heringdorf, R. van Gastei, B. Poelsema, and T. Michely, New J. Phys. 11, 039801 (2009).

${ }^{12}$ E. Sutter, P. Albrecht, and P. Sutter, Appl. Phys. Lett. 95, 133109 (2009)

${ }^{13}$ J. Wintterlin and M. L. Bocquet, Surf. Sci. 603, 1841 (2009).

${ }^{14}$ M. Treier, P. Ruffieux, R. Schillinger, T. Greber, K. Mullen, and R. Fasel, Surf. Sci. 602, L84 (2008).

${ }^{15}$ C. S. Shan, H. F. Yang, J. F. Song, D. X. Han, A. Ivaska, and L. Niu, Anal Chem. 81, 2378 (2009)

${ }^{16}$ Y. Y. Shao, J. Wang, H. Wu, J. Liu, I. A. Aksay, and Y. H. Lin, Electroanalysis 22, 1027 (2010).

${ }^{17}$ L. Wu, H. S. Chu, W. S. Koh, and E. P. Li, Opt. Express 18, 14395 (2010)

${ }^{18}$ H. Kanzow and A. Ding, Phys. Rev. B 60, 11180 (1999).

${ }^{19}$ S. Bhaviripudi, E. Mile, S. A. Steiner, A. T. Zare, M. S. Dresselhaus, A. M. Belcher, and J. Kong, J. Am. Chem. Soc. 129, 1516 (2007).

${ }^{20}$ D. N. Yuan, L. Ding, H. B. Chu, Y. Y. Feng, T. P. McNicholas, and J. Liu, Nano Lett. 8, 2576 (2008)

${ }^{21}$ N. Yoshihara, H. Ago, and M. Tsuji, Jpn. J. Appl. Phys. 47, 1944 (2008).

${ }^{22}$ V. L. De Los Santos, D. Lee, J. Seo, F. L. Leon, D. A. Bustamante, S. Suzuki, Y. Majima, T. Mitrelias, A. Ionescu, and C. H. W. Barnes, Surf. Sci. 603, 2978 (2009).

${ }^{23}$ X. Li, W. Cai, L. Colombo, and R. S. Ruoff, Nano Lett. 9, 4268 (2009).

${ }^{24}$ H. Okamoto and T. Massalski, J. Phase Equilibria 5, 378 (1984). 